\title{
Perioperative Statin Use and Acute Kidney Injury in Patients Undergoing Partial Nephrectomy
}

\author{
Shreyas S. Joshi*, Karen Ruth, Marc C. Smaldone, David Y.T. Chen, Richard E. Greenberg, \\ Rosalia Viterbo, Alexander Kutikov and Robert G. Uzzo \\ Department of Surgical Oncology, Division of Urologic Oncology, Fox Chase Cancer Center, Temple Health, \\ Philadelphia, PA, USA
}

\begin{abstract}
.
Background: Statin use is widespread among the general population. Data suggest a potentially beneficial effect of statin therapy on renal function following surgery. The impact of statins on post-partial nephrectomy (PN) renal function is unknown. We hypothesized that perioperative statin use may be associated with reduced rates of acute kidney injury (AKI) in patients undergoing PN.

Objectives: To evaluate the effect of perioperative statin use on AKI rates in patients undergoing PN.

Materials \& Methods: 1,056 patients undergoing PN were identified from a prospectively-maintained institutional renal mass database. Exclusion criteria included lack of preoperative serum creatinine $(\mathrm{Cr})$, concurrent surgeries, and those with baseline $\mathrm{Cr}<0.4$. The binary outcome was AKI, defined using modified Kidney Disease Improving Global Outcomes (KDIGO) criteria. Chi-Square and Cochran-Armitage trend tests were used to evaluate the strength of associations. A multivariate logistic regression model was used to determine predictors of AKI.

Results: Statin use was reported by $n=346$ (32.8\%) patients at the time of surgery. Univariate analysis demonstrated that statin use was associated with an increased risk of AKI following PN (OR 1.38, CI 1.01-1.88, $p=0.04$ ). On multivariate analysis, statin use was no longer associated with AKI following PN (OR 1.09, CI 0.76-1.56, $p=0.65$ ). Gender, BMI, comorbidity index, hypertension, surgical approach, ischemia temperature/time, and nephrometry " $\mathrm{R}$ " score were all independently associated with AKI.

Conclusions: Perioperative statin use at the time of PN was not associated with rates of post-operative AKI. Prospective studies are needed to elucidate the effects of statins on functional outcomes following PN.
\end{abstract}

Keywords: Statin, HMG-CoA reductase inhibitors, acute kidney injury, partial nephrectomy

\section{INTRODUCTION}

HMG-CoA reductase inhibitors (statins) have been shown to have a variety of beneficial medical effects and are associated with a reduced risk of all-cause and cardiovascular mortality and cardiovascular events [1]. Given their widespread use, the effect of

${ }^{*}$ Correspondence to: Shreyas S. Joshi, M.D., 333 Cottman Avenue, Philadelphia, PA 19111, USA. Tel.: +1 615924 1461; E-mail: Shreyas.joshi@fccc.edu. these medications on the surgical patient remains of interest. In the cardiac and neurosurgical literature, statin use has been studied based on outcomes related to a reduction in inflammatory biomarkers and possible effects on myocardial injury rates [2-4]. Since treatment of dyslipidemia can affect renovascular health [5], investigators have attempted to determine the renal effects of statin use in the perioperative setting. Two randomized controlled trials studying the role of statins on renal function in patients 
undergoing cardiac surgery were recently reported, but their conclusions were mixed [6,7].

Given their renovascular effects, one might expect statin use to have a measurable effect on renal function following ischemic cellular injury associated with partial nephrectomy (PN). Experimental models do show that statins can prevent ischemia-reperfusion injuries after renal ischemia [8]. The protective renovascular effects from statins stem from metabolic changes that protect against nitric oxide overproduction and promote endothelial cell stabilization by reducing oxidative stress within the glomerular microenvironment [9]. To date, the urologic literature regarding renal malignancies and statin use has focused on oncologic outcomes. In light of potential renal functional effects of statin use, we hypothesized that the use of statins might be associated with reduced rates of acute kidney injury (AKI) in patients undergoing PN.

\section{MATERIALS \& METHODS}

We queried our prospectively-assessed Fox Chase Cancer Center renal cancer database for patients undergoing any form of nephrectomy from January 2000 to May 2016. Of 2,312 eligible patients, we excluded patients who did not have a partial nephrectomy, who underwent multiple concurrent surgeries, had incomplete postoperative serum creatinine $(\mathrm{Cr})$ measurements, had a very low baseline serum $\mathrm{Cr}$ $(<0.4 \mathrm{mg} / \mathrm{dl})$, whose last recorded serum $\mathrm{Cr}$ was more than 120 days before surgery, and those whose last surgical consultation was $>6$ months prior to surgery (to avoid unrecorded changes in preoperative variables.) There were 1,056 patients undergoing PN who met the inclusion criteria (Fig. 1). Use of perioperative statins (yes/no) was determined by reviewing patient-reported pre-surgical medications. The dosage or duration of use was not included; patients could be taking more than one medication of this class. We did not have information on the indication for statin use.

AKI was the primary binary outcome and was defined using modified Kidney Disease Improving Global Outcomes (KDIGO) criteria [10]: An increase $\geq 1.5 \mathrm{x}$ baseline serum creatinine value occurring within 7 postoperative days, or an absolute increase of $\geq=0.3 \mathrm{mg} / \mathrm{dl}$ above baseline within the first 48 hours postoperatively. Urine output less than $0.5 \mathrm{~mL} / \mathrm{kg} / \mathrm{hr}$ is also a criterion, though could not be adequately assessed and was therefore omitted.
We compared patient characteristics by statin use, with Chi-square or Fisher's exact for categorical variables, and $t$-tests or Wilcoxon rank sum tests for continuous variables. Patient characteristics included patient demographics, smoking status, comorbidity score using the Charlson comorbidity index (CCI), diabetes, high cholesterol and hypertension status, elevated pre-surgical serum creatinine, and surgical procedure variables (type of surgery, ischemia type [warm/cold/none] and duration $[\leq=30 \mathrm{~min}$ vs $>30 \mathrm{~min}$ ], pathologic $\mathrm{T}$ stage). Nephrometry scores were available for 874 patients $(83 \%)$. For each patient characteristic, we assessed differences between categories in the AKI proportion using Chi-square tests and Cochran Armitage trend tests. We used multivariable logistic regression to adjust for potential confounders in the association between statin use and AKI, reporting the associations as odds ratios with $95 \%$ confidence intervals. To reduce the number of parameters in the multivariable model and avoid over-fitting, we included age at surgery, CCI, and BMI as continuous variables, after assessing the appropriateness of linear association. For 89 patients missing BMI data (8\%), we imputed values from the non-missing BMI patients based on a regression model for BMI with age and gender as predictors. As the CCI score includes diabetes and chronic kidney disease as conditions, we did not include these as separate covariates. We ran a separate multivariable model on the subset of patients with nephrometry scores ( $n=874$ patients, $83 \%$ ), which included components of the RENAL score to adjust for tumor complexity [11]. All analyses were performed using SAS statistical software (version 9.4, Cary NC).

\section{RESULTS}

Of the 1,056 patients included in this analysis, 670 (63\%) were male, and 911 (86.3\%) were Caucasian (Table 1). Mean age at surgery was 57.9 years, and mean BMI was $30.3 \mathrm{~kg} / \mathrm{m}^{2}$. One-third of patients $(\mathrm{N}=346,33 \%)$ reported using statin medications at the time of surgery. In this cohort, 218 patients $(21 \%)$ met the modified KIDGO definition of AKI following PN. Of the non-statin users, 134 patients (19\%) developed AKI, compared to 84 patients (24\%) who did use statins (Table 2).

As univariate analysis demonstrated, statin use was associated with an increased rate of postoperative AKI (OR 1.38, CI 1.01-1.88, $p=0.04$ ). Table 2 


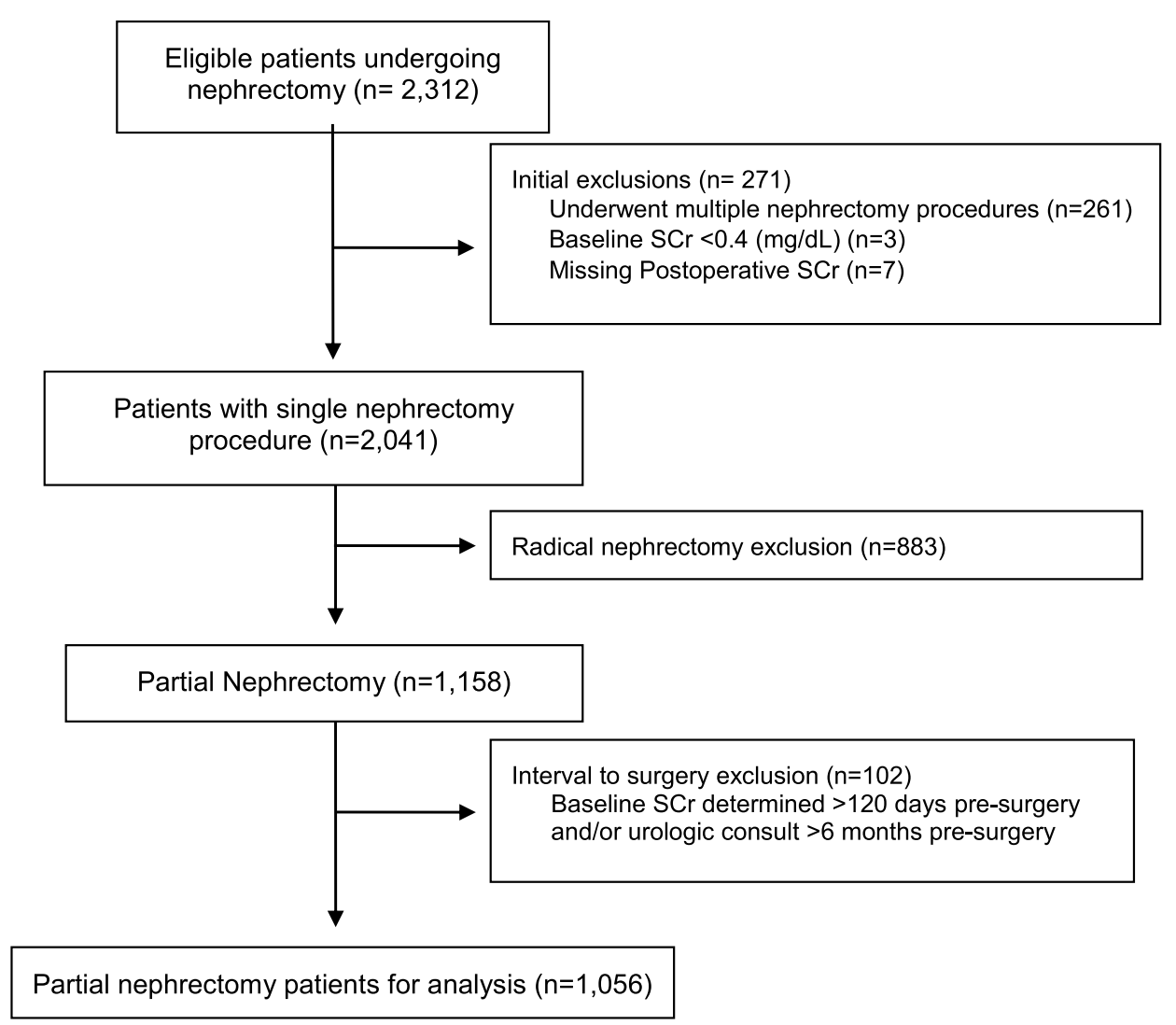

Fig. 1. Patient selection CONSORT diagram.

demonstrates all factors associated with AKI on univariate analysis. These included gender (male > female), BMI, weighted Charlson comorbidity index, hypertension, Type 1 and Type 2 diabetes, history of high cholesterol, pT stage, ischemia temperature, ischemia time (higher when $>30 \mathrm{~min}$ ), procedure approach (open $>$ robot/lap), composite nephrometry score (including specific associations with $\mathrm{R}, \mathrm{N}, \mathrm{A}$, and $\mathrm{L}$ components), baseline CKD stage, and elevated baseline serum $\mathrm{Cr}(40 \%$ with AKI for male $\mathrm{Cr}>1.4$ or female $\mathrm{Cr}>1.3$ vs. $19 \%$ with AKI for lower baseline $\mathrm{Cr}, p<0.001)$. AKI rates varied based on the diagnosis of hypercholesterolemia and based on the combination of statin use and the diagnosis of hypercholesterolemia (26.8\% of statin users with high cholesterol vs. $23.6 \%$ of statin users without high cholesterol, chi-square $p<0.01$ ).

After adjusting for significant covariates, statin use was no longer statistically associated with postoperative AKI (OR 1.09, CI 0.76-1.56, $p=0.65$ ). Factors that were independently associated with AKI (Table 3a) included gender (M vs F, OR 2.43, CI $1.57-3.75, p<0.001$ ), BMI (1 unit difference, OR
1.05 , CI $1.03-1.08, p<0.001)$, Charlson comorbidity index (OR 1.13, CI 1.01-1.27, $p=0.03$ ), history of hypertension (OR 1.68, CI 1.09-2.58, $p<0.001$ ), surgical approach (OR 2.73 for open vs. lap/robotic, CI 1.79-4.17 $p<0.001)$, and ischemia temperature/time, with duration of $>30 \mathrm{~min}$ (warm or cold) having higher likelihood of AKI compared to no ischemia $(p<0.001)$. A similar multivariable analysis was performed on the subset of patients with available nephrometry scores (Table 3b). In this model gender, BMI, hypertension, surgical approach, and ischemia temperature/time remained significant. However, Charlson comorbidity index did not remain significant. Nephrometry "R" (size) score was significantly associated with post-PN AKI $(4-7 \mathrm{~cm}$ vs $<4 \mathrm{~cm}$ OR 1.68, CI 1.11-2.55 $p=0.04)$.

\section{DISCUSSION}

Our data represent the most comprehensive single institutional cohort evaluating the effect of statins on post-PN AKI. By modified KDIGO criteria, the overall rate of AKI following PN was $20.6 \%$. We found 
Table 1

Patient characteristics by statin use $(n=1056)$

\begin{tabular}{|c|c|c|c|c|c|c|c|}
\hline \multirow[t]{2}{*}{ Characteristic } & \multicolumn{2}{|c|}{ All $(\mathrm{N}=1,056)$} & \multicolumn{2}{|c|}{$\begin{array}{l}\text { Statin Users } \\
(\mathrm{N}=346)\end{array}$} & \multicolumn{2}{|c|}{$\begin{array}{c}\text { Non-Users } \\
(\mathrm{N}=710) \\
\end{array}$} & \multirow[t]{2}{*}{$p$-value* } \\
\hline & Mean & StdDev & Mean & StdDev & Mean & StdDev & \\
\hline Age at Surgery, years & 57.9 & 11.7 & 62.5 & 9.5 & 55.7 & 12.0 & $<0.0001$ \\
\hline \multirow[t]{2}{*}{$\mathrm{BMI}, \mathrm{kg} / \mathrm{m}^{2} * *$} & 30.3 & 6.7 & 30.7 & 5.9 & 30.1 & 7.1 & 0.16 \\
\hline & $\mathrm{N}$ & Percent & $\mathrm{N}$ & Percent & $\mathrm{N}$ & Percent & \\
\hline Age at Surgery, years & & & & & & & $<0.0001$ \\
\hline $19-49$ & 243 & 23.0 & 30 & 8.7 & 213 & 30.0 & \\
\hline $50-59$ & 307 & 29.1 & 94 & 27.2 & 213 & 30.0 & \\
\hline $60-69$ & 348 & 33.0 & 145 & 41.9 & 203 & 28.6 & \\
\hline $70-89$ & 158 & 15.0 & 77 & 22.3 & 81 & 11.4 & \\
\hline Gender & & & & & & & $<0.0001$ \\
\hline Female & 386 & 36.6 & 90 & 26.0 & 296 & 41.7 & \\
\hline Male & 670 & 63.4 & 256 & 74.0 & 414 & 58.3 & \\
\hline Race & & & & & & & 0.46 \\
\hline White & 911 & 86.3 & 305 & 88.2 & 606 & 85.4 & \\
\hline Black/African American & 106 & 10.0 & 30 & 8.7 & 76 & 10.7 & \\
\hline Other & 39 & 3.7 & 11 & 3.2 & 28 & 3.9 & \\
\hline Smoking Status & & & & & & & 0.52 \\
\hline Current/Yes NOS & 161 & 15.2 & 51 & 14.7 & 110 & 15.5 & \\
\hline Former & 401 & 38.0 & 140 & 40.5 & 261 & 36.8 & \\
\hline No & 492 & 46.6 & 155 & 44.8 & 337 & 47.5 & \\
\hline Unknown & 2 & 0.2 & 0 & 0.0 & 2 & 0.3 & \\
\hline BMI category & & & & & & & 0.0039 \\
\hline Underweight $(<18.5)$ & 12 & 1.1 & 2 & 0.6 & 10 & 1.4 & \\
\hline Normal (18.5-24.9) & 174 & 16.5 & 46 & 13.3 & 128 & 18.0 & \\
\hline Overweight (25-29.9) & 343 & 32.5 & 112 & 32.4 & 231 & 32.5 & \\
\hline Obese (30-39.9) & 358 & 33.9 & 143 & 41.3 & 215 & 30.3 & \\
\hline Extremely Obese $(>=40)$ & 80 & 7.6 & 22 & 6.4 & 58 & 8.2 & \\
\hline Missing & 89 & 8.4 & 21 & 6.1 & 68 & 9.6 & \\
\hline Charlson Index & & & & & & & $<0.0001$ \\
\hline 0 (score is zero) & 541 & 51.2 & 138 & 39.9 & 403 & 56.8 & \\
\hline 1 to 2 & 350 & 33.1 & 137 & 39.6 & 213 & 30.0 & \\
\hline 3 to 4 & 127 & 12.0 & 50 & 14.5 & 77 & 10.8 & \\
\hline 5 to 9 & 38 & 3.6 & 21 & 6.1 & 17 & 2.4 & \\
\hline Hypertension & & & & & & & $<0.0001$ \\
\hline No & 432 & 40.9 & 87 & 25.1 & 345 & 48.6 & \\
\hline Yes & 624 & 59.1 & 259 & 74.9 & 365 & 51.4 & \\
\hline Type I DM & & & & & & & 0.33 \\
\hline No & 1013 & 95.9 & 329 & 95.1 & 684 & 96.3 & \\
\hline Yes & 43 & 4.1 & 17 & 4.9 & 26 & 3.7 & \\
\hline Type II DM & & & & & & & $<0.0001$ \\
\hline No & 904 & 85.6 & 258 & 74.6 & 646 & 91.0 & \\
\hline Yes & 152 & 14.4 & 88 & 25.4 & 64 & 9.0 & \\
\hline High Cholesterol & & & & & & & $<0.0001$ \\
\hline No & 643 & 60.9 & 71 & 20.5 & 572 & 80.6 & \\
\hline Yes & 413 & 39.1 & 275 & 79.5 & 138 & 19.4 & \\
\hline Baseline CKD stage & & & & & & & 0.0016 \\
\hline I & 427 & 40.4 & 119 & 34.4 & 308 & 43.4 & \\
\hline II & 476 & 45.1 & 164 & 47.4 & 312 & 43.9 & \\
\hline III & 147 & 13.9 & 61 & 17.6 & 86 & 12.1 & \\
\hline IV & 4 & 0.4 & 1 & 0.3 & 3 & 0.4 & \\
\hline $\mathrm{V}$ & 2 & 0.2 & 1 & 0.3 & 1 & 0.1 & \\
\hline
\end{tabular}

${ }^{*} p$-value is from $t$-test (continuous variables) or Chi-Square Test (categorical variables). ${ }^{* *}$ excludes 89 patients missing BMI at baseline (21 statin users, 68 non-users).

that increasing BMI, hypertension status, gender, Charlson comorbidity index, procedural approach, and ischemia temperature/time were associated with higher rates of AKI (Table 3a), as was nephrometry
"R" score (Table 3b). Perioperative statin use did not, however, associate with postoperative AKI after adjusting for covariates. These findings align with previously published work on smaller patient cohorts 
Table 2

Univariate associations with AKI after partial nephrectomy

\begin{tabular}{|c|c|c|c|c|}
\hline Characteristic & $\mathrm{N}$ & nAKI & $\%$ AKI & $p$-value \\
\hline All & 1056 & 218 & 20.6 & \\
\hline Age at Surgery (years) & & & & 0.21 \\
\hline $19-49$ & 243 & 39 & 16 & \\
\hline $50-59$ & 307 & 66 & 21.5 & \\
\hline $60-69$ & 348 & 75 & 21.6 & \\
\hline $70-89$ & 158 & 38 & 24.1 & \\
\hline Gender & & & & $<0.0001$ \\
\hline Female & 386 & 50 & 13 & \\
\hline Male & 670 & 168 & 25.1 & \\
\hline Race & & & & 0.3 \\
\hline White & 911 & 187 & 20.5 & \\
\hline Black & 106 & 26 & 24.5 & \\
\hline Other & 39 & 5 & 12.8 & \\
\hline Smoking Status & & & & 0.86 \\
\hline Current/Yes & 161 & 33 & 20.5 & \\
\hline Former & 401 & 86 & 21.4 & \\
\hline No & 492 & 99 & 20.1 & \\
\hline Unknown & 2 & 0 & 0 & \\
\hline BMI category & & & & 0.0016 \\
\hline Missing height or weight & 89 & 13 & 14.6 & \\
\hline Underweight $(<18.5)$ & 12 & 2 & 16.7 & \\
\hline Normal (18.5-24.9) & 174 & 28 & 16.1 & \\
\hline Overweight (25-29.9) & 343 & 60 & 17.5 & \\
\hline Obese $(30-39.9)$ & 358 & 87 & 24.3 & \\
\hline Extremely Obese $(>=40)$ & 80 & 28 & 35 & \\
\hline Weighted Charlson Index & & & & $<0.0001$ \\
\hline 0 (score is zero) & 541 & 90 & 16.6 & \\
\hline 1 to 2 & 350 & 73 & 20.9 & \\
\hline 3 to 4 & 127 & 38 & 29.9 & \\
\hline 5 to 9 & 38 & 17 & 44.7 & \\
\hline Hypertension & & & & $<0.0001$ \\
\hline No & 432 & 59 & 13.7 & \\
\hline Yes & 624 & 159 & 25.5 & \\
\hline Type I DM & & & & 0.0185 \\
\hline No & 1013 & 203 & 20 & \\
\hline Yes & 43 & 15 & 34.9 & \\
\hline Type II DM & & & & 0.0063 \\
\hline No & 904 & 174 & 19.2 & \\
\hline Yes & 152 & 44 & 28.9 & \\
\hline High Cholesterol & & & & 0.0057 \\
\hline No & 643 & 115 & 17.9 & \\
\hline Yes & 413 & 103 & 24.9 & \\
\hline Statin Use & & & & 0.0417 \\
\hline No & 710 & 134 & 18.9 & \\
\hline Yes & 346 & 84 & 24.3 & \\
\hline Baseline CKD Stage & & & & 0.0014 \\
\hline I & 427 & 82 & 19.2 & \\
\hline II & 476 & 88 & 18.5 & \\
\hline III & 147 & 44 & 29.9 & \\
\hline IV & 4 & 3 & 75 & \\
\hline $\mathrm{V}$ & 2 & 1 & 50 & \\
\hline Pathologic T Stage & & & & $<0.0001$ \\
\hline $\mathrm{T} 1 / 1 \mathrm{a} / 1 \mathrm{~b}$ & 938 & 175 & 18.7 & \\
\hline $\mathrm{T} 2 / 2 \mathrm{a} / 2 \mathrm{~b}$ & 57 & 24 & 42.1 & \\
\hline $\mathrm{T} 3 / 3 \mathrm{a} / 3 \mathrm{~b} / 3 \mathrm{c}$ & 54 & 18 & 33.3 & \\
\hline Tis/TX/miss & 7 & 1 & 14.3 & \\
\hline Serum Cr Baseline & & & & $<0.0001$ \\
\hline Male: $0.5-1.4$; Female $0.4-1.3$ & 970 & 184 & 19 & \\
\hline Male: $>1.4 ;$ Female $>1.3$ & 86 & 34 & 39.5 & \\
\hline
\end{tabular}

Table 2

(Continued)

\begin{tabular}{lcccc}
\hline Characteristic & $\mathrm{N}$ & nAKI & $\%$ AKI & $p$-value \\
\hline Procedure & & & & $<\mathbf{0 . 0 0 0 1}$ \\
$\quad$ Open & 446 & 143 & 32.1 & \\
$\quad$ Robotic/Laparoscopic & 610 & 75 & 12.3 & \\
Ischemia Type & & & & $<\mathbf{0 . 0 0 0 1}$ \\
$\quad$ Cold & 107 & 39 & 36.4 & \\
$\quad$ Warm & 747 & 146 & 19.5 & \\
$\quad$ N/A & 202 & 33 & 16.3 & \\
Ischemia Time & & & & $<\mathbf{0 . 0 0 0 1}$ \\
$\quad$ None & 202 & 33 & 16.3 & \\
$\quad<30$ min & 505 & 63 & 28.9 & \\
$\quad>30$ min & 349 & 122 & 56.0 &
\end{tabular}

RENL Nephrometry Score

$<0.0001$

(excludes 182 missing Neph score)

$\begin{array}{lccc}4-6 \text { (low) } & 245 & 28 & 11.4 \\ 7-9 \text { (moderate) } & 501 & 103 & 20.6 \\ 10-12 \text { (high) } & 128 & 45 & 35.2\end{array}$

10-12 (high) $\quad \begin{array}{lll}128 & 45 & 35.2\end{array}$

Largest Tumor $\mathrm{R}$

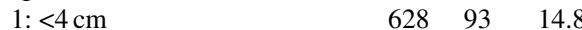

$2: 4-7 \mathrm{~cm} 202 \quad 65 \quad 32.2$

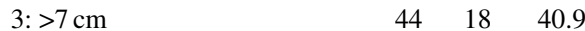

Largest Tumor E

1: $>50 \%$ exophytic $\quad 294 \quad 48 \quad 16.3$

2: $<50 \%$ exophytic $\quad 460 \quad 96 \quad 20.9$

3:100\% endophytic $\quad 120 \quad 32 \quad 26.7$

Largest Tumor $\mathrm{N}$

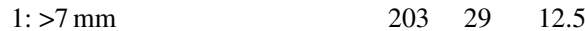

2:4-7 mm $\quad 96 \quad 21 \quad 18.0$

3: $<4 \mathrm{~mm} \quad 399 \quad 126 \quad 24.0$

Largest Tumor A

$\begin{array}{llll}\text { Anterior } & 383 & 61 & 15.9\end{array}$

$\begin{array}{llll}\text { Posterior } & 386 & 88 & 22.8\end{array}$

$\begin{array}{llll}\mathrm{X} \text { (neither anterior or posterior) } & 105 \quad 27 & 25.7\end{array}$

Largest Tumor L

$\begin{array}{llll}\text { Outside polar lines } & 288 & 41 & 14.2\end{array}$

$\begin{array}{llll}\text { Crosses polar line } & 281 & 69 & 24.6\end{array}$

Most/all within polar lines $\quad 305 \quad 66 \quad 21.6$

$<0.0001$

0.0501

0.0011

0.0188

0.0065

Univariate analysis showing variables associated with AKI follow-

ing PN for the entire PN cohort prior to exclusions $(\mathrm{N}=1056)$.

in which statin use had no association with AKI rates following PN [12].

On multivariate analysis, open surgery has a higher AKI rate than lap/robotic surgery. This may be, in part, due to the higher proportion of complex tumors removed via an open approach. Indeed, our data demonstrates that open surgery was associated with higher complexity tumors, with R.E.N.A.L. nephrometry scores of 10-12 (high-complexity) comprising $27.3 \%$ of open cases vs. only $7.2 \%$ of laparoscopic/robotic cases $(p<0.01)$. Of note, baseline CKD stage did associate with AKI on unadjusted analysis. The Charlson comorbidity index includes CKD as a component of its score, and it is notable that CCI was significant on multivariable analysis. Interestingly, this association failed to remain significant after controlling for nephrometry variables. The 
Table 3a

Multivariable logistic regression model for all patients $(n=1056$ patients with 218 AKI events)

\begin{tabular}{llrrr}
\hline & & OR est & $95 \%$ CI & p-value \\
\hline $\begin{array}{l}\text { Model 1*, unadjusted } \\
\text { Statin Use }\end{array}$ & Yes vs No & & & \\
Model 2*, Adjusted for Covariates & & 1.38 & $1.01-1.88$ & $\mathbf{0 . 0 4 2 2}$ \\
Statin Use & Yes vs No & & & \\
Gender & Male vs Female & 1.09 & $0.76-1.56$ & 0.6514 \\
BMI $(\mathrm{kg} / \mathrm{m} 2)$ & continuous & 2.47 & $1.67-3.66$ & $<\mathbf{0 . 0 0 0 1}$ \\
Charlson Comorbidity Index & continuous & 1.05 & $1.03-1.08$ & $<\mathbf{0 . 0 0 0 1}$ \\
Hypertension & Yes vs No & 1.13 & $1.01-1.27$ & $\mathbf{0 . 0 3 4 2}$ \\
Baseline serum Cr & High vs normal & 1.70 & $1.17-2.49$ & $\mathbf{0 . 0 0 6 0}$ \\
Pathologic T Stage & T2/2a/2b vs T1 & 1.40 & $0.78-2.50$ & 0.2573 \\
& T3/3a/3b/3c vs T1 & 1.47 & $0.77-2.79$ & 0.3091 \\
Surgical approach & Open vs Robot/Lap & 1.45 & $0.74-2.82$ & \\
Ischemia Temperature \& Time & Warm, <=30 min vs No ischemia & 3.08 & $2.12-4.48$ & $<\mathbf{0 . 0 0 0 1}$ \\
& Warm, $>30$ min vs No Ischemia & 3.45 & $0.64-1.81$ & $<\mathbf{0 . 0 0 0 1}$ \\
& Cold, $<=30$ min vs No Ischemia & 0.67 & $0.27-1.66$ & \\
& Cold, $>30$ min vs No Ischemia & 3.91 & $1.96-7.83$ & \\
\hline
\end{tabular}

${ }^{*} \mathrm{~N}=1056,218$ AKI events. Multivariate logistic regression evaluating predictors of AKI. Statin use was not an independent predictor of AKI when controlling for other variables.

Table $3 b$

Multivariable logistic regression model for patients with nephrometry scores ( $\mathrm{n}=874$ patients with 176 AKI events)

\begin{tabular}{|c|c|c|c|c|}
\hline & & \multicolumn{3}{|c|}{ Odds Ratio Estimates } \\
\hline & & OR est & $95 \% C I$ & $p$-value \\
\hline \multicolumn{5}{|l|}{ Model $1^{*}$, unadjusted } \\
\hline Statin Use & Yes vs No & 1.41 & $1.01-1.99$ & 0.0447 \\
\hline \multicolumn{5}{|l|}{ Model 2*, Adjusted for Covariates } \\
\hline Statin Use & Yes vs No & 1.12 & $0.75-1.67$ & 0.5892 \\
\hline Gender & Male vs Female & 2.43 & $1.57-3.75$ & $<0.0001$ \\
\hline BMI $(\mathrm{kg} / \mathrm{m} 2)$ & continuous & 1.05 & $1.02-1.08$ & 0.0006 \\
\hline Charlson Comorbidity Index & continuous & 1.10 & $0.96-1.26$ & 0.1578 \\
\hline Hypertension & Yes vs No & 1.68 & $1.09-2.58$ & 0.0180 \\
\hline Baseline serum $\mathrm{Cr}$ & High vs normal & 1.23 & $0.61-2.5$ & 0.5671 \\
\hline Surgical approach & Open vs Robot/Lap & 2.73 & $1.79-4.17$ & $<0.0001$ \\
\hline \multirow{4}{*}{ Ischemia Temperature \& Time } & Warm, $<=30 \mathrm{~min}$ vs No ischemia & 1.79 & $0.83-3.90$ & $<0.0001$ \\
\hline & Warm, $>30$ min vs No Ischemia & 4.92 & $2.28-10.62$ & \\
\hline & Cold, $<=30 \mathrm{~min}$ vs No Ischemia & 1.41 & $0.45-4.38$ & $<0.0001$ \\
\hline & Cold, >30 min vs No Ischemia & 7.95 & $3.09-20.44$ & \\
\hline \multirow[t]{2}{*}{ Nephrometry "R" Score } & $4-7 \mathrm{~cm}$ vs $<4 \mathrm{~cm}$ & 1.68 & $1.11-2.55$ & 0.0417 \\
\hline & $>7 \mathrm{~cm} \mathrm{vs}<4 \mathrm{~cm}$ & 1.60 & $0.75-3.42$ & \\
\hline \multirow[t]{2}{*}{ Nephrometry "A" Score } & Posterior vs Anterior & 1.55 & $1.03-2.33$ & 0.1128 \\
\hline & Neither vs Anterior & 1.29 & $0.72-2.32$ & \\
\hline \multirow[t]{2}{*}{ Nephrometry "L" Score } & Crosses polar line vs Outside polar lines & 1.37 & $0.84-2.22$ & 0.1187 \\
\hline & Mostly contained in polar lines vs Outside polar lines & 0.92 & $0.56-1.50$ & \\
\hline
\end{tabular}

${ }^{*} \mathrm{~N}=874$ patients with $176 \mathrm{AKI}$ events, includes those with nephrometry scores.

role of CKD on immediate post-PN renal function is therefore unclear in this analysis.

Statin use is very common in the United States, with a steady increase in use from $20 \%$ of all adults $>40$ yo in 2003 to nearly $30 \%$ in 2012 [13]. In large studies, statins have repeatedly been shown to have beneficial effects in patients with high cardiovascular risk $[1,3,14,15]$. A growing body of evidence appears to demonstrate that statins also have an impact on cancer biology.
Tumor cell proliferation and migration are cholesterol-dependent processes. Statins, by reducing the availability of circulating cholesterol, have been linked to cell-cycle interruption, repressed tumor cell proliferation, and impaired cell signaling [16-19]. These antitumor effects have been demonstrated in both metastatic RCC and after surgery for localized RCC [17, 19]. A recent meta-analysis by Nayan et al. evaluating statin use and kidney cancer survival outcomes showed that while statin use was 
not associated with recurrence-free or progressionfree survival, it was associated with significant improvements in cancer-specific (HR 0.67, 95\% CI $0.47-0.94$ ) and OS (HR $0.74,95 \%$ CI $0.63-0.88$ ) [20]. It is important to note, however, that the effect of statins on cancer mortality using observational data is subject to selection bias and immortaltime bias. Emilsson et al. recently demonstrated, by methodologically correcting for these biases, that previous observational designs may have overstated the relationships between statins and cancer mortality [21].

The protective renovascular effects from statins can be mediated by metabolic changes, such as decreases in nitric oxide overproduction, which have been shown in animal models to stabilize endothelial cells and reduce oxidative stress [9]. Such vasculoprotective drug effects motivated investigation into the potential role of statins on renovascular outcomes following major elective surgery. Some large retrospective studies did find a positive impact of statins on postoperative AKI rates [22, 23], while others did not [24]. The renovascular effects of statins have been most extensively studied following cardiac surgery. The results of these investigations are mixed; large retrospective series appear to show a protective effect from statin use, while more recent prospective randomized data show a potential negative effect on renovascular function $[6,25]$. A meta-analysis involving 23 randomized controlled trials and $>5,000$ patients also demonstrated an increased risk of AKI with statin use in this population (OR 1.26, 95\% CI 1.05-1.52) [4]. Several RCTs have subsequently concluded that statins likely have no measurable impact on postoperative AKI following cardiac surgery [7, 26].

The theoretical impact of statins following PN relate to the ischemic risks related to the procedure. Operative ischemia and preoperative atherosclerotic disease are considered risk factors for postoperative AKI; and as such, statins might play a role in decreasing the injurious effect of inflammation and oxidative stress within the kidney [27]. Indeed, HMG-CoA reductase has been demonstrated within the glomerular and peritubular microvasculature of normal kidneys; and statins have been experimentally used to prevent ischemia-reperfusion injury in rat kidney transplant models [8]. Despite these plausible mechanisms, the present study did not find a measurable association between statin use postoperative AKI following PN.

The reported rates of AKI following PN vary due to the inconsistent classification of AKI and reliance on administrative datasets. Several nationwide studies report very low AKI rates following PN. For example, data from the National Surgical Quality Improvement Program (NSQIP) found that a combined $1.8 \%$ of patients following radical/partial nephrectomy developed postoperative AKI, but the definition of AKI was an elevation in serum $\mathrm{Cr}$ $>2 \mathrm{mg} / \mathrm{dl}$ above baseline or need for dialysis, which are less stringent than KDIGO criteria [28]. Schmid et al. evaluated the National Inpatient Sample (maintained by the Agency for Healthcare Research and Quality) of 253,000 patient and found a postoperative AKI rate following PN of approximately 5\% [29]. Our results, on the other hand, demonstrated a 20.6\% AKI rate using the more comprehensive KDIGO criteria.

A limitation of this analysis is its retrospective nature and reliance on patient-reported statin use. Additionally, the study was not controlled for dose or duration; hence the impact of long- vs. short-term statin use on AKI rates may be a topic for further investigation. We also do not have perioperative lipid profiles, hence cannot know if statin use had its intended therapeutic effects. This analysis could not control for other medications that may influence postoperative renal function, such as ACE-inhibitors. Any mild effects of statins on postoperative AKI therefore could have been camouflaged by competing pharmacologic factors. Long-term renal functional outcomes are of particular interest in this population, which the KDIGO criteria do not comprehensively capture. Unfortunately, we were unable to sufficiently report on 6-12 month renal functional outcomes due to database limitations, though we would aim to report on such outcomes in future investigations. Given the diversity of patient comorbidities, perioperative variables, and the impact of renal mass complexity on a multitude of postoperative outcomes, a prospective trial may ultimately be needed to fully elucidate the relationship between statin medications and renal functional outcomes following PN.

\section{CONCLUSION}

Although statin medication use has previously demonstrated a renoprotective effect in patients undergoing cardiac surgery, more recent evidence from the cardiac and non-cardiac surgical literature indicates that this effect may not be reproducible. Using a large retrospective patient cohort, we did not identify any association between statin use 
and the risk of post-PN AKI after adjustment for clinicopathologic variables. Although some evidence suggests that statins improve oncologic outcomes in $\mathrm{RCC}$, there does not appear to be a clear renal functional impact of perioperative statin use. Prospective, controlled analysis may be needed to uncover further effects of statins on post-PN outcomes.

\section{ACKNOWLEDGMENTS}

The authors thank Debra Kister and Michelle Collins for statistical and database support for this study.

\section{FINANCIAL DISCLOSURE}

None of the authors have any relevant financial disclosures to report.

\section{CONFLICT OF INTEREST}

None of the authors have any relevant conflicts of interest to report.

\section{FUNDING}

This publication was supported in part by grant number P30 CA006927 from the National Cancer Institute. Its contents are solely the responsibility of the authors and does not necessarily represent the official views of the National Cancer Institute or the National Institutes of Health. Cases were reviewed from the Fox Chase Cancer Center Prospectively Maintained Kidney Data Base.

\section{DATA ACCESS/DATA ANALYSIS}

We, Shreyas Joshi and Karen Ruth, had full access to all the data in the study and take responsibility for the integrity of the data and the accuracy of the data analysis.

\section{REFERENCES}

[1] Chou R, Dana T, Blazina I, et al. Statin Use for the Prevention of Cardiovascular Disease in Adults: A Systematic Review for the US Preventive Services Task Force. Rockville (MD), 2016.

[2] Chello M, Patti G, Candura D, et al. Effects of atorvastatin on systemic inflammatory response after coronary bypass surgery. Crit Care Med. 2006;34:660-7.
[3] Shepherd J, Cobbe SM, Ford I, et al. Prevention of coronary heart disease with pravastatin in men with hypercholesterolemia. West of Scotland Coronary Prevention Study Group. N Engl J Med. 1995:333:1301-7.

[4] Putzu A, Capelli B, Belletti A, et al. Perioperative statin therapy in cardiac surgery: A meta-analysis of randomized controlled trials. Crit Care. 2016;20:395.

[5] Balafa O, Kalaitzidis R, Siamopoulos KC. Optimal medical management in patients with renovascular hypertension. Am J Cardiovasc Drugs. 2013;13:71-8.

[6] Zheng Z, Jayaram R, Jiang L, et al. Perioperative Rosuvastatin in Cardiac Surgery. N Engl J Med. 2016;374:1744-53.

[7] Billings FTt, Hendricks PA, Schildcrout JS, et al. High-dose perioperative atorvastatin and acute kidney injury following cardiac surgery: A randomized clinical trial. JAMA. 2016;315:877-88

[8] Tuuminen R, Nykanen AI, Saharinen P, et al. Donor simvastatin treatment prevents ischemia-reperfusion and acute kidney injury by preserving microvascular barrier function. Am J Transplant. 2013;13:2019-34.

[9] Giusti-Paiva A, Martinez MR, Felix JV, et al. Simvastatin decreases nitric oxide overproduction and reverts the impaired vascular responsiveness induced by endotoxic shock in rats. Shock. 2004;21:271-5.

[10] Khwaja A. KDIGO clinical practice guidelines for acute kidney injury. Nephron Clin Pract. 2012;120:c179-84.

[11] Kutikov A, Uzzo RG. The R.E.N.A.L. nephrometry score: A comprehensive standardized system for quantitating renal tumor size, location and depth. J Urol. 2009;182:844-53.

[12] Krane LS, Sandberg JM, Rague JT, Hemal AK. Do statin medications impact renal functional or oncologic outcomes for robot-assisted partial nephrectomy? J Endourol. 2014;28:1308-12.

[13] Gu Q, Paulose-Ram R, Burt VL, Kit BK. Prescription cholesterol-lowering medication use in adults aged 40 and over: United States, 2003-2012. NCHS Data Brief. 2014:1-8.

[14] Nielsen SF, Nordestgaard BG, Bojesen SE. Statin use and reduced cancer-related mortality. N Engl J Med. 2013;368:576-7.

[15] Davies JT, Delfino SF, Feinberg CE, et al. Current and Emerging Uses of Statins in Clinical Therapeutics: A Review. Lipid Insights. 2016;9:13-29.

[16] Boudreau DM, Yu O, Johnson J. Statin use and cancer risk: A comprehensive review. Expert Opin Drug Saf. 2010;9:603-21.

[17] McKay RR, Lin X, Albiges L, et al. Statins and survival outcomes in patients with metastatic renal cell carcinoma. Eur J Cancer. 2016;52:155-62.

[18] Haddad AQ, Jiang L, Cadeddu JA, et al. Statin use and serum lipid levels are associated with survival outcomes after surgery for renal cell carcinoma. Urology. 2015;86:1146-52.

[19] Kaffenberger SD, Lin-Tsai O, Stratton KL, et al. Statin use is associated with improved survival in patients undergoing surgery for renal cell carcinoma. Urol Oncol. 2015;33:21 e11-7.

[20] Nayan M, Punjani N, Juurlink DN, et al. Statin use and kidney cancer survival outcomes: A systematic review and meta-analysis. Cancer Treat Rev. 2017;52:105-16.

[21] Emilsson L, Garcia-Albeniz X, Logan RW, Caniglia EC, Kalager M, Hernan MA. Examining bias in studies of statin treatment and survival in patients with cancer. JAMA Oncol. 2018;4:63-70

[22] Molnar AO, Coca SG, Devereaux PJ, et al. Statin use associates with a lower incidence of acute kidney injury 
after major elective surgery. J Am Soc Nephrol. 2011;22: 939-46.

[23] Brunelli SM, Waikar SS, Bateman BT, et al. Preoperative statin use and postoperative acute kidney injury. Am J Med. 2012;125:1195-204 e3

[24] Argalious MY, Dalton JE, Sreenivasalu T, O'Hara J, Sessler DI. The association of preoperative statin use and acute kidney injury after noncardiac surgery. Anesth Analg. 2013; 117:916-23.

[25] Layton JB, Kshirsagar AV, Simpson RJ, Jr., et al. Effect of statin use on acute kidney injury risk following coronary artery bypass grafting. Am J Cardiol. 2013;111:823-8.

[26] Park JH, Shim JK, Song JW, Soh S, Kwak YL. Effect of atorvastatin on the incidence of acute kidney injury following valvular heart surgery: A randomized, placebo-controlled trial. Intensive Care Med. 2016;42:1398-407.

[27] Abdul Rahman MN, Chetter IC. Statins and ischaemia reperfusion injury: A molecular biological review. Curr Vasc Pharmacol. 2010;8:831-5.

[28] Schmid M, Abd-El-Barr AE, Gandaglia G, et al. Predictors of 30-day acute kidney injury following radical and partial nephrectomy for renal cell carcinoma. Urol Oncol. 2014;32:1259-66.

[29] Schmid M, Krishna N, Ravi P, et al. Trends of acute kidney injury after radical or partial nephrectomy for renal cell carcinoma. Urol Oncol. 2016;34:293 e1-e10. 